\title{
EDITORIAL
}

\section{CAMBIO DE LA DENOMINACIÓN DE LAS ESCUELAS UNIVERSITARIAS DE ENFERMERÍA A FACULTADES DE ENFERMERÍA}

\author{
Carmen Isabel Gómez García. \\ Enfermera. Profesora Titular de Universidad. \\ Directora Escuela de Enfermería Universidad de Murcia
}

Constituye para mí un honor y un gran motivo de satisfacción el hecho de haber sido invitada por el Profesor Dr. José Siles a escribir esta editorial en un momento tan importante para las Escuelas de Enfermería por múltiples motivos entre los que se encuentran la consecución del cambio de la denominación de Escuelas Universitarias a Facultades de Enfermería.

Los estudios de Enfermería, en nuestro país, podríamos decir que son privilegiados ya que han conseguido a lo largo del tiempo y en su desarrollo una apreciación y reconocimiento social manifestado en la gran demanda de los mismos debido a la calidad de sus enseñanzas y larga tradición. La primera referencia legal que encontramos sobre la formación de personal sanitario, se encuentra en la Ley de Instrucción Pública de 9 de septiembre de 1857, que en sus artículos 40 y 41 hace mención a los títulos de practicante y matrona o partera. La primera Escuela de Enfermería de España (Santa Isabel de Hungría), se crea en el año 1896, pero no es hasta 1955 cuando se crea el título de Ayudante Técnico Sanitario que unifica, de una parte, el título de enfermera reconocido en la Real Orden del 7 de mayo de 1915 y, de otro lado, los títulos de practicante y matrona establecidos por la Ley de Instrucción Pública (mencionada anteriormente) del 9 de Septiembre de 1857, más conocida como la Ley Moyano. Es en 1977 cuando los estudios de Enfermería se reconocen como universitarios siguiendo las directrices del Consejo de Europa (77/453/CEE).

La Escuela de Enfermería de la Universidad de Murcia se creó en el año 1991 en virtud de la sus- cripción de los convenios de colaboración entre la Universidad de Murcia, el Instituto Nacional de la Salud del que dependía la antigua Escuela de Enfermería "Virgen de la Arrixaca" y la Universidad de Murcia con la Comunidad Autónoma de la que dependía la "Escuela de la Comunidad Autónoma", aprobados en Junta de Gobierno (7 de Mayo de 1990) y ratificados por el Consejo Social de la misma, estas Escuelas venían funcionando desde los años 60 y como fruto de su unión se inician los estudios de enfermería dentro del marco de una Escuela propia de la Universidad de Murcia a partir del curso académico 1991/1992 y según las cláusulas de los respectivos convenios. B.O.E. 12 de octubre de 1991 (creación de centros) $)^{1}$.

De modo paralelo a la integración y desarrollo de los estudios conducentes a la obtención de la Diplomatura en Enfermería, el compromiso institucional de la Universidad de Murcia con la titulación ha sido patente, ya que ha promovido los estudios de postgrado y las estructuras necesarias para ello. En el año 2001 se crearon tanto la Unidad Docente Obstétrico-Ginecológica (Matrona) como la Escuela de Práctica Enfermera, de modo que los egresados por esta Universidad pueden seguir cursando estudios de posgrado.

Asimismo el profesorado ha impartido docencia en distintos masteres y posgrados de Enfermería en universidades españolas tales como la de Alicante, Huelva, Almería o la Autónoma de Madrid, Granada, La Laguna, Jaén y Málaga, en nuestra propia Universidad, y universidades extranjeras a través de programas internacionales.

${ }^{\prime}$ Tomado del Título de Grado de Enfermería por la Universidad de Murcia 
Si cualitativamente la Escuela de Enfermería ha realizado un esfuerzo meridiano en la mejora continua de su Plan Formativo y relaciones institucionales a nivel nacional e internacional, la capacidad formativa de este centro está avalada cuantitativamente por el alto número de egresados que finalizan todos los años sus estudios.

Considerando que la situación académica actual de la Escuela de Enfermería de la Universidad de Murcia presenta unas características idóneas en la temporalidad debido a la aprobación del Título de Grado en Enfermería y su inicio en el curso académico 2009-2010, con un total de 238 alumnos, la aprobación del Máster oficial en Salud, Mujer y Cuidados iniciado su impartición también en este curso 2009-2010, la aprobación del Sistema de Garantía Interna de Calidad y la incorporación a la homologación del Título a Graduados desde Diplomados en Enfermería de 35 egresados.

Destacar también que la propuesta del Programa de doctorado en Enfermería denominado: Investigación en Cuidados de Enfermería por la Universidad de Murcia, fue concebido y estructurado desde el rigor y la experiencia que la disciplina enfermera viene demostrando desde finales del siglo XX en que nos incorporamos a la universidad española, ofertando líneas referidas a: Factores relacionados con el derecho a la salud de la mujer en el mundo, Estudio de los diagnósticos enfermeros desde la perspectiva de género, Cuidados enfermeros creativos, Epidemias comunitarias: promoción de la salud, Prevención y cuidados, Clínica del cuidado enfermero a la infancia y adolescencia, Puericultura enfermera, Malos tratos en la infancia, Lactancia materna, Formación enfermera en Europa, Enfermería y discapacidad en los distintos grupos de edad, Historia de la Enfermería, Historia de las mujeres, Historia de las parteras, filosofía y ciencia de la enfermería, Calidad de vida y envejecimiento, Redes de cuidados, Cuidado informal, Psicología forense, Salud laboral, Administración y gestión de calidad de los servicios de salud, Factores psicológicos relacionados con la salud laboral y la gestión de recursos humanos, Humanización en la asistencia al embarazo, parto y puerperio de la mujer, La carrera profesional en Enfermería, Enfermería, actividad física y deportiva para la salud, Mujer, ciencia y cul- tura, Mujer y cuidados en la edad media y renacimiento, Mujeres y enfermería, Promoción y cuidados en salud de la mujer, Violencia de género, Salud mental y mujer, Calidad de vida relacionada con la salud, Estudio de los problemas asociados a los resultados de salud mediante encuestas, Aplicación y evaluación de la práctica clínica basada en la evidencia, Epidemiología y tratamiento de los trastornos del sueño, Enfermería y partería en la Organización Mundial de la Salud y por último La ética del cuidado en la asistencia sanitaria, siendo todas estas líneas de investigación ofertadas por profesores del Área de conocimiento de Enfermería, departamento de Enfermería.

La denominación del Centro ha dependido de los estudios oficiales que estamos autorizados a impartir y existe una jerarquía entre Facultades y Escuelas Universitarias incluso desde la perspectiva de la imagen que la sociedad puede tener de los distintos Centros y que la vigente Ley Orgánica de Universidades, en su artículo 7 señala que "Las universidades públicas estarán integradas por Escuelas, Facultades, Departamentos, Institutos Universitarios de Investigación y por aquellos otros centros o estructuras necesarios para el desempeño de sus funciones". Y en el artículo siguiente indica que "Las escuelas y facultades son los centros encargados de la organización de las enseñanzas y de los procesos académicos, administrativos y de gestión conducentes a la obtención de títulos de grado. Podrán impartir también enseñanzas conducentes a la obtención de otros títulos, así como llevar a cabo aquellas otras funciones que determine la universidad".

Como se puede observar en este texto, desaparecen las denominaciones de "Escuelas Técnicas Superiores" y "Escuelas Universitarias" y se incorpora como nombre de centro las "Escuelas".

De este precepto se deduce de forma inmediata que los actuales centros deben adaptar su denominación a las previsiones de la Ley, sólo pudiendo existir "Facultades" o "Escuelas". No cabe, bajo la vigencia de la actual ley, la persistencia de las Escuelas Universitarias.

Una correcta interpretación de la norma, que podía haber previsto expresamente la transformación de las Escuelas Técnicas Superiores y de las Escuelas Universitarias, y teniendo en cuenta los 
datos y argumentos hasta aquí expuestos, pasa por considerar que la Ingenierías y Arquitecturas se imparten en "Escuelas" y el resto de grados en Facultades.

Podríamos añadir como argumentos, por un lado, que estos centros ya pueden impartir posgrados oficiales y doctorado y, por otro, que ha desaparecido la figura del Profesor Titular de Escuela Universitaria porque todos los profesores titulares pueden impartir docencia en todos los grados y posgrados.

Por último, en el caso de las Escuelas Universitarias de Enfermería, de las que son predicables todos los argumentos esgrimidos, cabe añadir que se homologarían a nuestros centros internacionales, dónde es común la denominación de "Facultades de Enfermería".

Según el proverbio chino de que las grandes cosas se explican con palabras sencillas, me gustaría dejar constancia que mis compañeros miembros del Consejo de Gobierno de la Universidad de Murcia, decidieron por unanimidad secundar la propuesta del Consejo de Dirección (Equipo Rectoral) presentada por el Sr. Secretario General Dr. José Fulgencio Angosto, apoyando el punto 4. del orden del día del Consejo de Gobierno de 22 de enero de 2010, que rezaba así: Aprobación, si procede, de la adecuación de la denominación de los centros de la Universidad de Murcia a la Ley 4/2007 Orgánica de Universidades. Por lo que tras su aprobación, fue remitido al órgano superior Consejo Social de la Universidad de Murcia y tratado el día 30 de abril de 2010, obteniendo aquí esta misma propuesta presentada por el Rector Dr. José Antonio Cobacho, el apoyo unánime de los componentes de dicho órgano. Quedando pendiente el visto bueno de la Comunidad Autónoma tras la aportación de la documentación requerida a dicha propuesta con la justificación de la misma, datos académicos, datos demográficos, demanda de las titulaciones, informes de infraestructura, informes de satisfacción de egresados, perfiles profesionales, apoyos externos y datos referidos al Sistema de Garantía Interna de Calidad.

Esta editorial no puede contentarse con una narración más o menos extensa de la situación vivida por mucho que en el desarrollo de la misma haya sido considerado por los actores de sumo interés y absolutamente imprescindibles. Debe sustentarse en una documentación escrita fruto del esfuerzo, la no pasividad y de ser conscientes de que en nuestro paso por la gestión se debe visualizar el compromiso de renovar y actualizar nuestras obligaciones esenciales para con el sistema educativo, en unas enseñanzas queridas y valoradas por la sociedad que respeta y reconoce las acciones proactivas de los Cuidados de Enfermería y por ende de la profesión enfermera.

Para finalizar, como quiera que no se trata de la creación de un nuevo centro, sino de la modificación de su denominación para la adaptación a las prescripciones que sobre esa materia establece la LOU, consideramos que esta modificación debe ser automática, mediante acuerdo de los órganos universitarios y autonómicos competentes, constituyendo una oportunidad única para Enfermería en el momento de hacer emerger los apoyos Académicos, Sociales y Políticos que nos ayudan en nuestro crecimiento como Disciplina. 